\title{
Retrospective review of the activity and safety of apatinib and anlotinib in patients with advanced osteosarcoma and soft tissue sarcoma
}

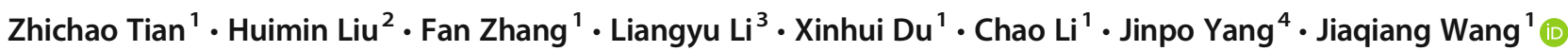

Received: 4 December 2019 / Accepted: 12 February 2020 / Published online: 25 February 2020

(C) The Author(s) 2020

\section{Summary}

Background Previous studies have demonstrated the efficacy of apatinib and anlotinib for the treatment of sarcomas. However, more clinical data and evidence are needed to support clinical treatment selection and study design. Here, we evaluated the effectiveness and safety of these two drugs for the treatment of sarcomas. Methods We retrospectively reviewed the data of 110 patients with advanced osteosarcoma $(n=32)$ or soft tissue sarcoma (STS, $n=78)$ who received oral apatinib or anlotinib therapy during May 2016-February 2019 at two centers. Patients were divided into the apatinib and anlotinib groups. Results Among osteosarcoma patients, the objective response rates (ORRs) for the apatinib and anlotinib groups were 15.79\% (3/19) and 7.69\% (1/13), respectively. The disease control rates (DCRs) were 63.16\% (12/19) and 30.77\% (4/13), and the median progression-free survival (m-PFS) was $4.67 \pm 3.01$ and $2.67 \pm 1.60$ months, respectively. Among STS patients, ORRs for the apatinib and anlotinib groups were $12.24 \%$ (6/49) and 13.79\% (4/29), respectively. The DCRs were 59.18\% (29/49) and 55.17\% (16/29), and m-PFS was $7.82 \pm 6.90$ and $6.03 \pm 4.50$ months, respectively. Regarding adverse events (AEs), apatinib was associated with a higher incidence of hair hypopigmentation and pneumothorax, while anlotinib was associated with a higher incidence of pharyngalgia or hoarseness. Conclusion Both apatinib and anlotinib were effective for the treatment of sarcomas. However, the effectiveness of the two drugs and associated AEs varied based on the histological type of sarcoma. These differences may be due to their different sensitivities to targets such as RET, warranting further study.

Keywords Tyrosine kinase inhibitor · Osteosarcoma $\cdot$ Soft tissue sarcoma $\cdot$ Adverse events $\cdot$ Pneumothorax

\section{Introduction}

Sarcomas are malignancies of mesenchymal origin, of which more than 70 histological subtypes have been identified [1-3].

Jiaqiang Wang

doctorwangjiaqiang@126.com

1 Department of Orthopedics, The Affiliated Cancer Hospital of Zhengzhou University and Henan Cancer Hospital,

Zhengzhou 450008, Henan Province, China

2 Department of Medical Oncology, The Affiliated People's Hospital of Zhengzhou University, Zhengzhou 450003, Henan Province, China

3 Department of Orthopedics, the People's Hospital of Jiyuan, Jiyuan 459000, Henan Province, China

4 Department of Medical Oncology, The Affiliated Cancer Hospital of Zhengzhou University and Henan Cancer Hospital,

Zhengzhou 450008, Henan Province, China
Sarcomas are relatively rare, with an annual incidence of fewer than 5 cases per 100,000 people, and these malignancies account for $1-2 \%$ of all adult cancers $[1,3]$. Despite the rarity of sarcomas, more than 20,000 new cases of sarcoma are diagnosed annually in China [4]. Sarcomas can be subdivided into bone tumors (mostly osteosarcoma) and soft tissue sarcomas (STSs) according to pathological and histological features [5].

Surgical resection is the most important treatment option for sarcomas. However, 30-50\% of sarcomas eventually recur or metastasize after surgery, and some patients present with metastases at the initial diagnosis $[6,7]$. For metastatic or locally unresectable cases, doxorubicin-based chemotherapy is the first-line treatment. However, the response rates of advanced sarcomas to chemotherapy are only $14-48 \%[8,9]$. Therefore, more effective clinical treatments for advanced sarcomas are needed.

The emergence of small-molecule, multi-target tyrosine kinase inhibitors (TKIs) has advanced the treatment of sarcoma. Since the initial approval of pazopanib for STSs by the United 
States Food and Drug Administration (FDA) in 2012 [10], an increasing number of studies have demonstrated the efficacy of TKIs for the treatment of sarcomas [11, 12]. Apatinib is a smallmolecule drug that potently and highly selectively inhibits the tyrosine kinase activity of vascular endothelial growth factor receptor 2 (VEGFR2) in vitro, and also inhibits the activities of VEGFR1, Kit, c-SRC, and RET tyrosine kinases [13]. This drug was approved by the Chinese FDA (CFDA) for the treatment of advanced gastric cancer in 2014 [14]. Anlotinib is a newly developed oral small-molecule TKI that targets VEGFR2, VEGFR3, Kit, VEGFR1, platelet-derived growth factor receptor (PDGFR)- $\alpha$, and multiple fibroblast growth factor receptors (FGFR1, FGFR2, and FGFR3) [15]. This drug was approved by the CFDA for the treatment of advanced non-small cell lung cancer in 2018 [16].

Studies have revealed that apatinib and anlotinib display promising activity against sarcomas [17-19]. As the Affiliated People's Hospital of Zhengzhou University and Affiliated Cancer Hospital of Zhengzhou University are two major sarcoma treatment centers in central China, we have treated many advanced sarcoma patients with multi-target TKIs. For several reasons, some patients were treated with apatinib, while others were treated with anlotinib. In this study, we retrospectively investigated these patients and studied the similarities and differences between patients treated with apatinib and anlotinib, with the aim of providing more evidence to support clinical treatment selection and clinical study design.

\section{Methods}

\section{Patients and eligibility criteria}

This was a retrospective study of patients treated at two hospitals: Affiliated People's Hospital of Zhengzhou University and Affiliated Cancer Hospital of Zhengzhou University. The study was performed according to the principles and guidelines of the Declaration of Helsinki and approved by the Institutional Review Board of the Affiliated Cancer Hospital of Zhengzhou University. Patient enrollment began in May 2016 and ended in February 2019. The patient eligibility criteria included the following: 1) histologically proven osteosarcoma or STS; 2) age between 15 and 70 years; 3) confirmed ineligibility for radiotherapy or surgical treatment; 4) Eastern Cooperative Oncology Group Performance Status (ECOG PS) 0 or 1 ; 5) no history of treatment with other targeted drugs; 6) measurable lesions according to the Response Evaluation Criteria in Solid Tumors (RECIST) 1.1; and 7) acceptable hematologic (absolute neutrophil count $\geq 1.5 \times 10^{9}$ cells $/ \mathrm{L}$, platelets $\geq 100 \times 10^{9} / \mathrm{L}$, and hemoglobin concentration $\geq$ $9 \mathrm{~g} / \mathrm{dL}$ ); hepatic (aspartate aminotransferase and alanine aminotransferase $\leq 2.5 \times$ upper limit of normal [ULN], bilirubin $\leq 1.5 \times \mathrm{ULN}$, and alkaline phosphatase $\leq 2.5 \times$ $\mathrm{ULN}$ ); and renal function (serum creatinine $\leq 1.5 \times$ ULN, glomerular filtration rate $\geq 30 \mathrm{~mL} / \mathrm{min}$ per $1.73 \mathrm{~m}^{2}$ according to the modified diet in renal disease abbreviated formula, and normal spot urine analysis results). This analysis was considered descriptive, and follow-up was extended until November 30, 2019.

\section{Treatment}

According to the received treatment, patients were divided into the apatinib and anlotinib groups. In the apatinib group, patients received a once-daily oral dose of $500 \mathrm{mg}$ apatinib. This apatinib dose was reduced to $250 \mathrm{mg}$ per day for patients with intolerable adverse events (AEs). In the anlotinib group, patients received a once-daily oral dose of $12 \mathrm{mg}$ anlotinib on days 1-14 of a 21-day cycle. This anlotinib dose was reduced to $10 \mathrm{mg}$ per day for patients with intolerable AEs. Both drugs were administered continuously until intolerable AEs or progressive disease (PD) occurred. AEs were assessed using the National Cancer Institute Common Terminology Criteria for Adverse Events (NCICTCAE), version 4.0. If a severe AE occurred, apatinib or anlotinib administration was delayed for a maximum of 14 days to enable recovery.

\section{Evaluation}

Tumor responses were evaluated every 2 months with computed tomography or magnetic resonance imaging. If a clear signal of PD was observed, evaluation was performed immediately. Tumor responses were evaluated according to the RECIST version 1.1 and were categorized as a complete response (CR), partial response (PR), stable disease (SD), or PD. The objective response rate (ORR) was defined as the sum of the rates of $\mathrm{CR}$ and PR. The disease control rate (DCR) was defined as the sum of the ORR and SD. Differences in the ORR, DCR, median progression-free survival (m-PFS), and AE incidence between the anlotinib and apatinib groups were also assessed. PFS was calculated from the date of the first dose of apatinib or anlotinib until the date of documented progression or death from any cause.

\section{Statistical analysis}

Quantitative variables are presented as medians (ranges) or numbers of patients (percentages). PFS was estimated using the Kaplan-Meier method with a $95 \%$ confidence interval (CI). The survival curves were generated using GraphPad Prism 5.0 (La Jolla, CA, USA). Statistical analyses were 
Table 1 Basic characteristics of the two osteosarcoma groups

\begin{tabular}{|c|c|c|}
\hline Characteristics & Apatinib group $(n=19)$ & Anlotinib group $(n=13)$ \\
\hline \multicolumn{3}{|l|}{ Gender } \\
\hline Male & $10(52.63 \%)$ & $5(38.46 \%)$ \\
\hline Female & $9(47.37 \%)$ & $8(61.54 \%)$ \\
\hline Age & $22.42 \pm 13.26$ & $20.46 \pm 11.15$ \\
\hline \multicolumn{3}{|l|}{ ECOG PS } \\
\hline 0 & $11(57.89 \%)$ & $7(53.85 \%)$ \\
\hline 1 & $8(42.11 \%)$ & $6(46.15 \%)$ \\
\hline \multicolumn{3}{|l|}{ Primary site } \\
\hline Femur & $6(31.58 \%)$ & $5(38.46 \%)$ \\
\hline Axial skeleton & $2(10.53 \%)$ & $1(7.69 \%)$ \\
\hline Tibia & $5(26.32 \%)$ & $4(30.77 \%)$ \\
\hline Humerus & $3(15.79 \%)$ & $2(15.38 \%)$ \\
\hline Fibula & $1(5.26 \%)$ & $0(0.00 \%)$ \\
\hline Other & $1(5.26 \%)$ & $1(7.69 \%)$ \\
\hline Radial & $1(5.26 \%)$ & $0(0.00 \%)$ \\
\hline \multicolumn{3}{|c|}{ Excision of primary lesion } \\
\hline No & $3(15.79 \%)$ & $2(15.38 \%)$ \\
\hline Yes & $16(84.21 \%)$ & $11(84.62 \%)$ \\
\hline \multicolumn{3}{|l|}{ Metastatic site } \\
\hline Only lung & $14(73.68 \%)$ & $10(76.92 \%)$ \\
\hline Only bone & $1(5.26 \%)$ & $1(7.69 \%)$ \\
\hline Both bone and lung & $4(21.05 \%)$ & $2(15.38 \%)$ \\
\hline \multicolumn{3}{|c|}{ Previous MAP/I chemotherapy } \\
\hline No & $1(5.26 \%)$ & $0(0.00 \%)$ \\
\hline Yes & $18(94.74 \%)$ & $13(100.00 \%)$ \\
\hline \multicolumn{3}{|c|}{ Previous other chemotherapy } \\
\hline No & $17(89.47 \%)$ & $12(92.31 \%)$ \\
\hline Yes & $2(10.53 \%)$ & $1(7.69 \%)$ \\
\hline Time interval (months) & $4.32 \pm 2.81$ & $4.62 \pm 2.40$ \\
\hline
\end{tabular}

Notes: Data are presented as numbers (percentages) or means \pm standard deviations

Abbreviations: ECOG PS, Eastern Cooperative Oncology Group performance status; MAP/I, high-dose methotrexate, doxorubicin, cisplatin, and/or ifosfamide; Time interval, time interval between the end of chemotherapy and oral apatinib or anlotinib administration performed using SPSS 21.0 software for Windows (IBM Corp., Armonk, NY, USA).

\section{Results}

\section{Patient characteristics}

A total of 121 patients received apatinib or anlotinib treatment during the study period. Eight patients were lost to follow-up; three patients dropped out for other reasons. Finally, $110 \mathrm{pa}-$ tients were enrolled, including 32 with osteosarcoma and 78 with STS.

The characteristics of the osteosarcoma patients are shown in Table 1. Nineteen and 13 patients received apatinib and anlotinib, respectively, and these groups had average ages of $22.42 \pm 13.26$ and $20.46 \pm 11.15$ years, respectively. Among patients in the apatinib group, $57.89 \%$ and $42.11 \%$ had an ECOG PS of 0 or 1, respectively. Among patients in the anlotinib group, $53.85 \%$ and $46.15 \%$ had an ECOG PS of 0 or 1, respectively. The primary lesions were distributed all over the body, although the most common sites were the femur, tibia, humerus, and axial skeleton. Most patients underwent primary lesion excision surgery $(84.21 \%$ [16/19] and $84.62 \%$ $[11 / 13]$ patients in the apatinib and anlotinib groups, respectively). The lung was the most frequent location of metastases (94.74\% [18/19] and 92.31\% [12/13] patients in the apatinib and anlotinib groups, respectively). The average elapsed time from the end of chemotherapy to 
Table 2 Basic characteristics of the two soft tissue sarcoma groups

\begin{tabular}{|c|c|c|}
\hline Characteristics & Apatinib group $(n=49)$ & Anlotinib group $(n=29)$ \\
\hline \multicolumn{3}{|l|}{ Gender } \\
\hline Male & $27(55.10 \%)$ & $15(51.72 \%)$ \\
\hline Female & $22(44.90 \%)$ & $14(48.28 \%)$ \\
\hline Age & $41.10 \pm 14.20$ & $41.86 \pm 14.27$ \\
\hline \multicolumn{3}{|l|}{ ECOG PS } \\
\hline 0 & $24(48.98 \%)$ & $15(51.72 \%)$ \\
\hline 1 & $25(51.02 \%)$ & $14(48.28 \%)$ \\
\hline \multicolumn{3}{|l|}{ Histological type } \\
\hline UPS & $10(20.41 \%)$ & $5(17.24 \%)$ \\
\hline Synovial sarcoma & $7(14.29 \%)$ & $7(24.14 \%)$ \\
\hline Leiomyosarcoma & $6(12.24 \%)$ & $3(10.34 \%)$ \\
\hline Liposarcoma & $5(10.20 \%)$ & $2(6.90 \%)$ \\
\hline MPNST & $4(8.16 \%)$ & $1(3.45 \%)$ \\
\hline Angiosarcoma & $4(8.16 \%)$ & $1(3.45 \%)$ \\
\hline Clear cell sarcoma & $3(6.12 \%)$ & $0(0.00 \%)$ \\
\hline Epithelioid sarcoma & $3(6.12 \%)$ & $2(6.90 \%)$ \\
\hline Rhabdomyosarcoma & $3(6.12 \%)$ & $1(3.45 \%)$ \\
\hline Fibrosarcoma & $2(4.08 \%)$ & $3(10.34 \%)$ \\
\hline ASPS & $1(2.04 \%)$ & $3(10.34 \%)$ \\
\hline Malignant granulosa cell tumor & $1(2.04 \%)$ & $0(0.00 \%)$ \\
\hline PNET & $0(0.00 \%)$ & $1(3.45 \%)$ \\
\hline \multicolumn{3}{|l|}{ Locally unresectable or metastatic } \\
\hline Locally unresectable & $7(14.29 \%)$ & $5(17.24 \%)$ \\
\hline Metastatic & $42(85.71 \%)$ & $24(82.76 \%)$ \\
\hline \multicolumn{3}{|l|}{ Primary site } \\
\hline Extremities & $34(69.39 \%)$ & $20(68.97 \%)$ \\
\hline Trunk & $15(30.61 \%)$ & $9(31.03 \%)$ \\
\hline \multicolumn{3}{|l|}{ Excision of primary lesion } \\
\hline No & $8(16.33 \%)$ & $4(13.79 \%)$ \\
\hline Yes & $41(83.67 \%)$ & $25(86.21 \%)$ \\
\hline \multicolumn{3}{|l|}{ Metastatic site } \\
\hline Lungs & $43(87.76 \%)$ & $25(86.21 \%)$ \\
\hline Other & $6(12.24 \%)$ & $4(13.79 \%)$ \\
\hline \multicolumn{3}{|l|}{ Lines of previous chemotherapy } \\
\hline 1 & $33(67.35 \%)$ & $17(58.62 \%)$ \\
\hline 2 & $12(24.49 \%)$ & $11(37.93 \%)$ \\
\hline 3 & $4(8.16 \%)$ & $1(3.45 \%)$ \\
\hline Time interval (months) & $4.78 \pm 2.04$ & $4.55 \pm 2.21$ \\
\hline
\end{tabular}

Notes: Data are presented as numbers (percentages) or means \pm standard deviations

Abbreviations: ECOG PS, Eastern Cooperative Oncology Group performance status; UPS, undifferentiated pleomorphic sarcoma; MPNST, malignant peripheral nerve sheath tumor; ASPS, alveolar soft part sarcoma; PNET, primitive neurotodermal tumor; Time interval, time interval between the end of chemotherapy and oral apatinib or anlotinib administration the start of TKI treatment was $4.32 \pm 2.81$ and $4.62 \pm$ 2.40 months in the apatinib and anlotinib groups, respectively.

The basic characteristics of the STS patients are listed in Table 2. Forty-nine and 29 patients received apatinib and anlotinib, respectively. The average ages were 41.10 \pm 14.20 and $41.86 \pm 14.27$ years in the apatinib and anlotinib groups, respectively. All patients had a good performance status (ECOG PS 0/1). The histological subtypes included undifferentiated pleomorphic sarcoma 
Table 3 Clinical efficacy of apatinib and anlotinib in osteosarcoma

\begin{tabular}{lll}
\hline Characteristics & Apatinib group $(\mathrm{n}=19)$ & Anlotinib group $(\mathrm{n}=13)$ \\
\hline ORR (\%) & $3(15.79 \%)$ & $1(7.69 \%)$ \\
DCR (\%) & $12(63.16 \%)$ & $4(30.77 \%)$ \\
m-PFS (months) & $4.67 \pm 3.01$ & $2.67 \pm 1.60$ \\
\hline
\end{tabular}

Notes: Data are presented as numbers (percentages) or means \pm standard deviations

Abbreviations: ORR, objective response rate; DCR, disease control rate; $\mathrm{m}$-PFS, median progression-free survival

(UPS, $n=15)$; synovial sarcoma $(n=14)$; leiomyosarcoma $(n=9)$; liposarcoma $(n=7)$; malignant peripheral nerve sheath tumor $(n=5)$; angiosarcoma $(n=5)$; epithelioid sarcoma $(\mathrm{n}=5)$; rhabdomyosarcoma $(n=4)$; fibrosarcoma $(\mathrm{n}=5)$; alveolar soft part sarcoma $(n=3)$; clear cell sarcoma $(\mathrm{n}=3)$; malignant granulosa cell tumor $(n=1)$; and primitive neuroectodermal tumor $(\mathrm{n}=1)$. Most patients underwent primary lesion excision surgery $(83.67 \%$ [41/ 49] patients in the apatinib group and $86.21 \%$ [25/29] patients in the anlotinib group). The lung was the most frequent site of metastasis $(87.76 \%$ [43/49] patients in the apatinib group and $86.21 \%$ [25/29] patients in the anlotinib group), and all patients had received at least one cycle of chemotherapy previously. The average elapsed time from the end of chemotherapy to the start of TKI treatment was $4.78 \pm 2.04$ and $4.55 \pm 2.21$ months in the apatinib and anlotinib groups, respectively.

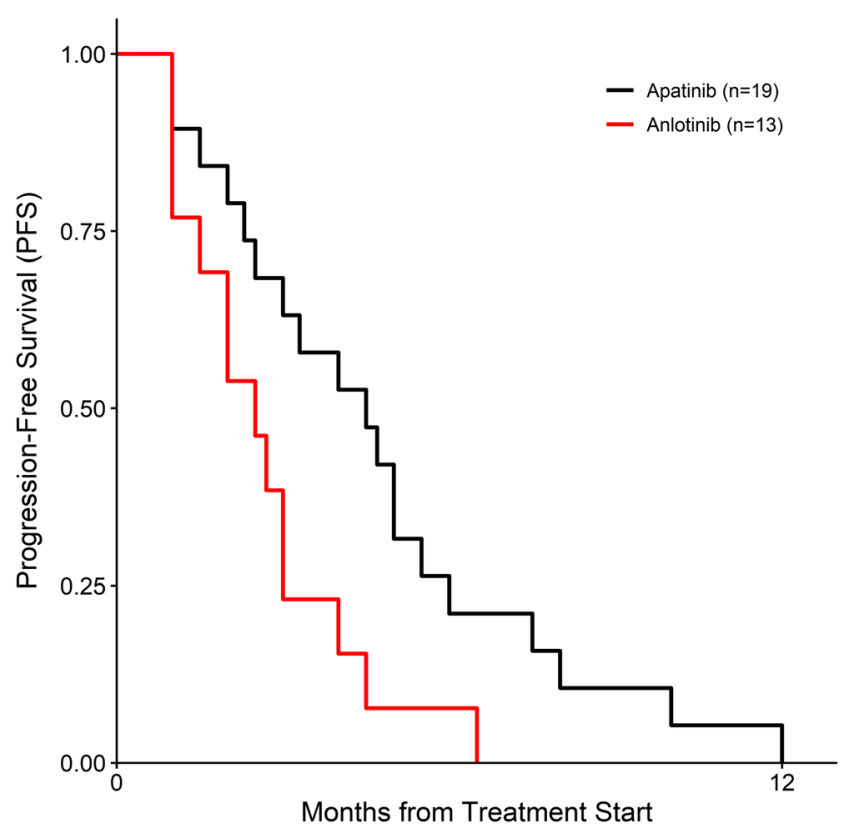

Fig. 1 Kaplan-Meier estimates of progression-free survival among patients with osteosarcoma after treatment with apatinib or anlotinib

\section{Clinical effectiveness}

\section{Osteosarcoma patients}

None of the 32 osteosarcoma patients achieved CR. The apatinib group had an ORR of $15.79 \%$, DCR of $63.16 \%$, and $\mathrm{m}$-PFS of $4.67 \pm 3.01$ months. The anlotinib group had an ORR of $0.00 \%$, DCR of $23.08 \%$, and m-PFS of $2.67 \pm$ 1.60 months (Table 3, Fig. 1).

\section{STS patients}

One UPS patient in the apatinib group achieved a CR (Table 4). The apatinib group had an ORR of $12.24 \%$, DCR of $59.18 \%$, and m-PFS of $7.82 \pm 6.90$ months. The anlotinib group had an ORR of $13.79 \%$, DCR of $55.17 \%$, and m-PFS of $6.03 \pm 4.50$ months (Table 5, Fig. 2).

\section{Toxicity evaluation}

AEs appeared to be more prevalent in the apatinib group than in the anlotinib group (Table 6). Most AEs were grade 1 or 2 , although a few were grade 3 or 4, and no drug-related deaths occurred. Some AEs occurred more frequently in the apatinib group, including hair hypopigmentation and pneumothorax.

Table 4 Responses of various histological subtypes to treatment

\begin{tabular}{|c|c|c|c|c|c|c|c|c|}
\hline \multirow[t]{2}{*}{ Histological subtype } & \multicolumn{4}{|c|}{ Apatinib group $(n=49)$} & \multicolumn{4}{|c|}{ Anlotinib group $(n=29)$} \\
\hline & $\mathrm{CR}$ & PR & $\mathrm{SD}$ & $\mathrm{PD}$ & $\mathrm{CR}$ & PR & $\mathrm{SD}$ & $\mathrm{PD}$ \\
\hline UPS & 1 & 3 & 4 & 2 & & 1 & 1 & 3 \\
\hline Synovial sarcoma & & 1 & 4 & 2 & & 1 & 4 & 2 \\
\hline Leiomyosarcoma & & 1 & 2 & 3 & & 1 & 2 & \\
\hline Liposarcoma & & & 3 & 2 & & & 1 & 1 \\
\hline MPNST & & & 1 & 3 & & & & 1 \\
\hline Angiosarcoma & & 1 & 2 & 1 & & & & 1 \\
\hline Clear cell sarcoma & & & 1 & 2 & & & & \\
\hline Epithelioid sarcoma & & & 1 & 2 & & & 1 & 1 \\
\hline Rhabdomyosarcoma & & & 2 & 1 & & & & 1 \\
\hline Fibrosarcoma & & & 1 & 1 & & & 1 & 2 \\
\hline ASPS & & 1 & & & & 1 & 2 & \\
\hline MGCT & & & & 1 & & & & \\
\hline PNET & & & & & & & & 1 \\
\hline Total & 1 & 7 & 21 & 20 & & 4 & 12 & 13 \\
\hline
\end{tabular}

Abbreviations: $\mathrm{CR}$, complete response; $\mathrm{PR}$, partial response; SD, stable disease; PD, progressive disease; UPS, undifferentiated pleomorphic sarcoma; MPNST, malignant peripheral nerve sheath tumor; ASPS, alveolar soft part sarcoma; MGCT, malignant granulosa cell tumor; PNET, primitive neurotodermal tumor 
Table 5 Clinical efficacy of apatinib and anlotinib in soft tissue sarcoma

\begin{tabular}{lll}
\hline Characteristics & Apatinib group $(\mathrm{n}=49)$ & Anlotinib group $(\mathrm{n}=29)$ \\
\hline ORR (\%) & $6(12.24 \%)$ & $4(13.79 \%)$ \\
DCR (\%) & $29(59.18 \%)$ & $16(55.17 \%)$ \\
m-PFS (months) & $7.82 \pm 6.90$ & $6.03 \pm 4.50$ \\
\hline
\end{tabular}

Notes: Data are presented as numbers (percentages) or means \pm standard deviations

Abbreviations: ORR, objective response rate; DCR, disease control rate; $\mathrm{m}$-PFS, median progression-free survival

Pharyngalgia or hoarseness was more frequent in the anlotinib group (Table 6).

\section{Discussion}

The treatment of advanced sarcomas and efficacies of therapies have long remained stagnant. The current fourdrug combination chemotherapy regimen was established as the first-line treatment for advanced osteosarcoma in the 2000s [7] and is associated with m-PFS of $<4$ months [12]. For advanced STS, doxorubicin-based chemotherapy has been administered as the first-line treatment for decades [6], and is associated with m-PFS of 5-8 months [9]. However, this scenario has changed with the advent of TKIs. TKIs target tyrosine kinases, which are key mediators of intracellular signaling cascades. Consequently,

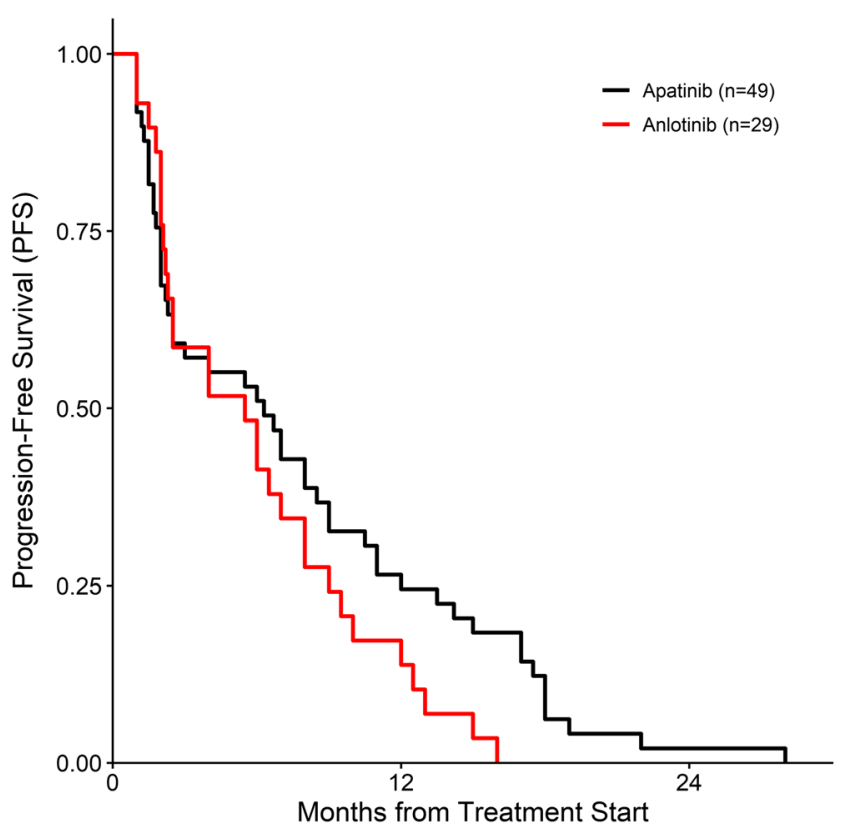

Fig. 2 Kaplan-Meier estimates of progression-free survival among patients with soft tissue sarcoma after treatment with apatinib or anlotinib aberrations in these proteins have been implicated as drivers of oncogenesis via the dysregulation of fundamental cellular processes, including proliferation, migration, and apoptosis [20]. TKI-based therapy has led to significant advances in the treatment of many malignancies. All TKIs with promising preclinical and clinical effectiveness against sarcoma, including apatinib, anlotinib, axitinib, imatinib, pazopanib, regorafenib, sorafenib, and sunitinib, target multiple angiogenic and growth-promoting receptor tyrosine kinases, as shown in Table 7.

Apatinib and anlotinib, the only two domestically developed multi-target TKIs, have been marketed and used widely for the treatment of advanced sarcomas in China [17, 18, 21]. Although many clinical studies of apatinib and anlotinib have begun to recruit patients with sarcoma (http://www.chictr.org.cn), this study is the first to simultaneously investigate the effectiveness and safety of these two drugs in patients with advanced sarcomas. In this retrospective observational study, we found that both apatinib and anlotinib were effective for the treatment of sarcomas. However, the effectiveness of the two drugs and corresponding AEs varied based on the histological type of sarcoma. Apatinib appeared to be more effective in osteosarcoma, and it was associated with higher incidences of hair hypopigmentation and pneumothorax. On the other hand, anlotinib was associated with a higher incidence of pharyngalgia or hoarseness.

Our finding that apatinib was effective for the treatment of osteosarcoma was consistent with the results of previous studies. To date, at least five studies have demonstrated the effectiveness of apatinib for treatment of osteosarcoma [17, 22-25]. While none have reported the effectiveness of anlotinib. Although both drugs are multi-target TKIs, they differ with regard to the therapeutic effectiveness against osteosarcoma. We screened clinical trials registered with ClinicalTrials.gov for nearly all smallmolecule TKIs and found that only three TKIs (apatinib, regorafenib, and sorafenib) have been identified as promising for the treatment of osteosarcoma $[12,22,26]$. These three TKIs share a distinctive sensitivity for VEGFR2 and RET (Tables 7 and 8) [13, 27-30], suggesting that RET, like VEGFR2, may be an important specific target in the treatment of osteosarcoma.

In contrast, we did not observe a difference in the therapeutic effects of apatinib and anlotinib in patients with STS. Several other studies have also demonstrated the effectiveness of both TKIs for the treatment of STS [19, 21, 25, 31, 32]. However, these two TKIs differ with respect to therapeutic effectiveness for specific subtypes of STS. For example, we demonstrated different effectiveness of these drugs for the treatment of UPS and leiomyosarcoma (Table 4). Nonetheless, the large number of STS subtypes falsely suggests that these two TKIs have similar efficacies. We speculate that these different therapeutic effects of apatinib and anlotinib on different subtypes of STS involve a fundamental difference in target sensitivity (Table 8). 


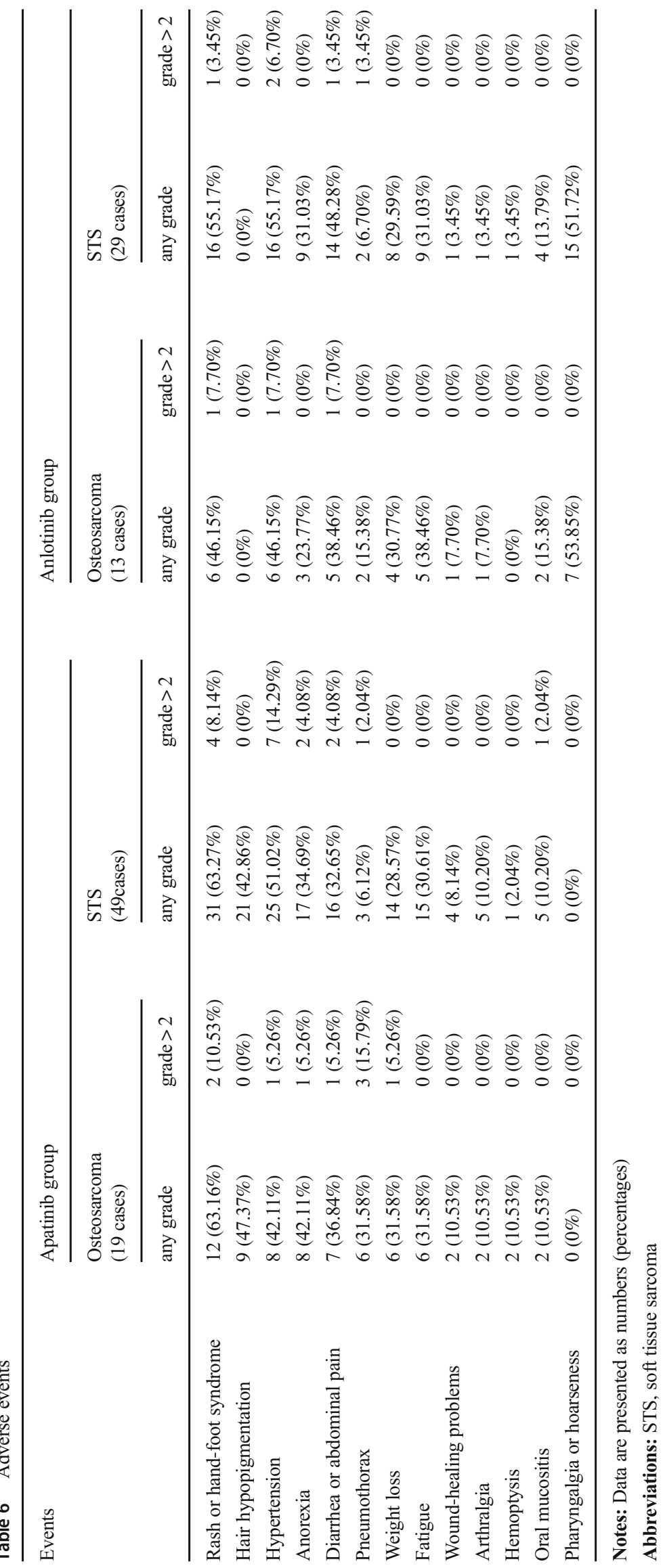




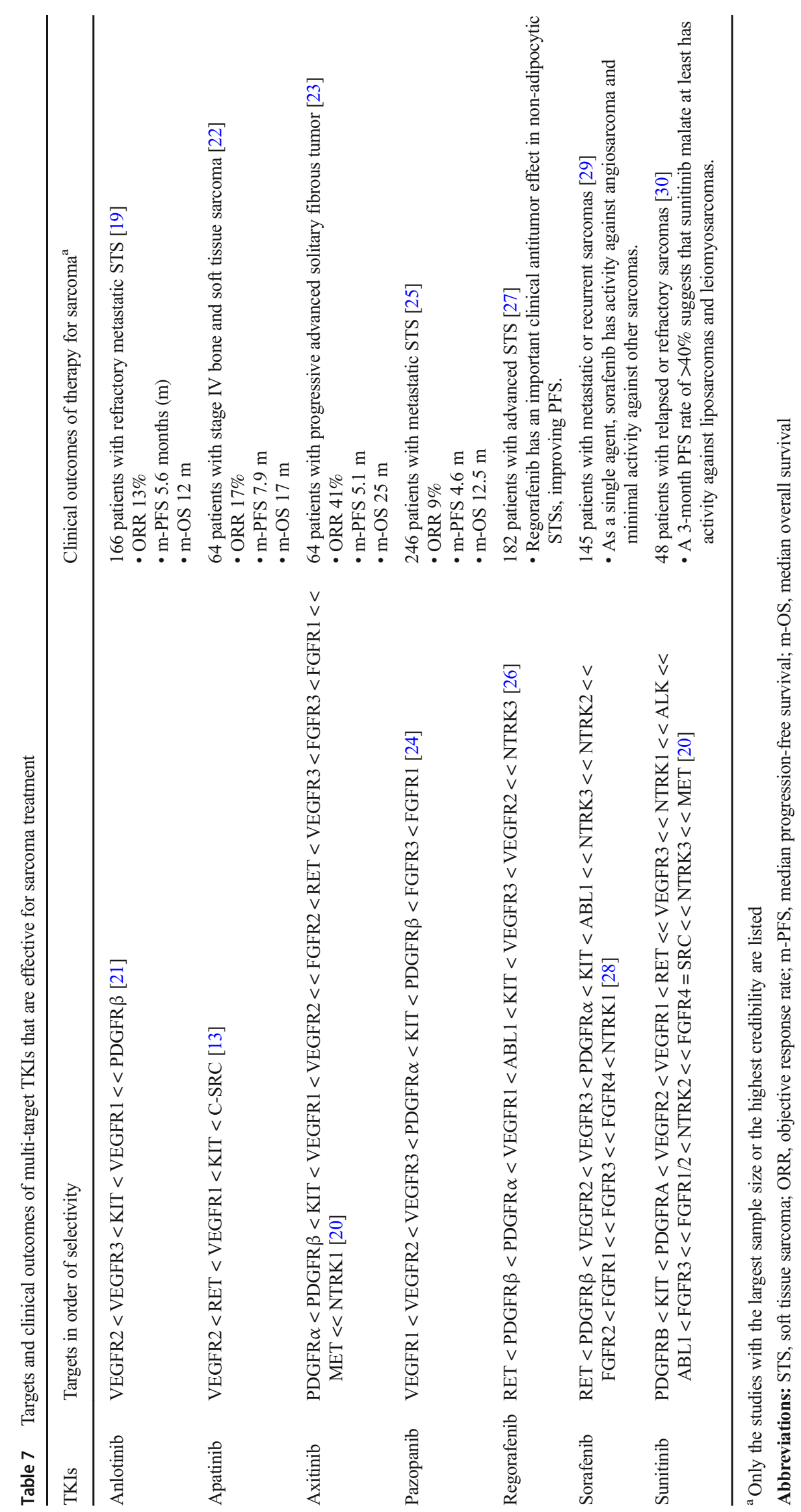


Table 8 Sensitive targets of anlotinib, apatinib, sorafenib, and regorafenib

\begin{tabular}{lllll}
\hline Kinases & \multicolumn{2}{l}{$\mathrm{IC}_{50}(\mathrm{nM}$, mean $)$} \\
\cline { 2 - 5 } & Anlotinib & Apatinib & Sorafenib & Regorafenib \\
\hline VEGFR1 & 26.9 & 70 & - & 13 \\
VEGFR2 & 0.2 & 1 & 4 & 4.2 \\
VEGFR3 & 0.7 & - & 20 & 46 \\
KIT & 14.8 & 429 & 68 & 7 \\
PDGFR $\alpha$ & 167 & $>1000$ & 57 & 22 \\
RET & n.d. & 13 & 0.4 & 1.5 \\
C-SRC & - & 530 & - & - \\
FGFR1 & 40.4 & - & 580 & 202 \\
\hline
\end{tabular}

Abbreviations: $\mathrm{IC}_{50}$, half maximal inhibitory concentration; $\mathrm{nM}, \mathrm{nmol} / \mathrm{l}$; n.d., not determined

Further research is required to elucidate the mechanisms of action beyond the currently identified targets.

We further observed an increased incidence of pneumothorax in patients with osteosarcoma who were treated with apatinib rather than with anlotinib. In such cases, the basic pathological process of pneumothorax involved necrosis, cavitation in the metastatic lung lesions, and finally pneumothorax formation (as shown in Fig. 3), consistent with other reports of osteosarcoma $[31,32]$. However, apatinib was not reported to induce this AE in patients with other types of malignancies (i.e., non-sarcoma) [14, 33]. Moreover, significant increases in pneumothorax were not observed in response to sorafenib and regorafenib, which are as effective as apatinib for osteosarcoma [12,26]. We also observed that the incidences of hair hypopigmentation and pharyngalgia or hoarseness differed between the apatinib and anlotinib groups.
We speculate that these differences could be attributable to the targets of these TKIs (Table 8).

The main limitations of this study include the retrospective design, the absence of a control group, and the abundance of uncommon sarcoma subtypes. A registered clinical study on the efficacy of apatinib versus anlotinib for different subtypes of sarcoma must be conducted to obtain more accurate and reliable evidence. Moreover, the mechanism by which apatinib induces pneumothorax during the treatment of pulmonary metastatic osteosarcoma requires further study. More importantly, studies on the role and mechanism of RET in the treatment of osteosarcoma by multi-target TKIs may yield unexpected results.

In conclusion, apatinib and anlotinib were both effective for the treatment of sarcomas. The effectiveness of the two drugs and associated AEs varied based on the histological type of sarcoma. These differences may be due to their different sensitivities to targets such as RET, warranting further study.

Acknowledgements We are grateful to all the patients and investigators in this study. This research did not receive any specific grant from funding agencies in the public, commercial, or not-for-profit sectors.

Funding information This research did not receive any specific grant from funding agencies in the public, commercial, or not-for-profit sectors.

\section{Compliance with ethical standards}

Conflict of interest All authors who took part in this study indicate that they do not have anything to declare regarding funding or conflicts of interest with respect to this study.

Ethics approval All procedures performed in studies involving human participants were in accordance with the ethical standards of the institutional and/or national research committee and with the 1964 Helsinki Declaration and its later amendments or comparable ethical standards.

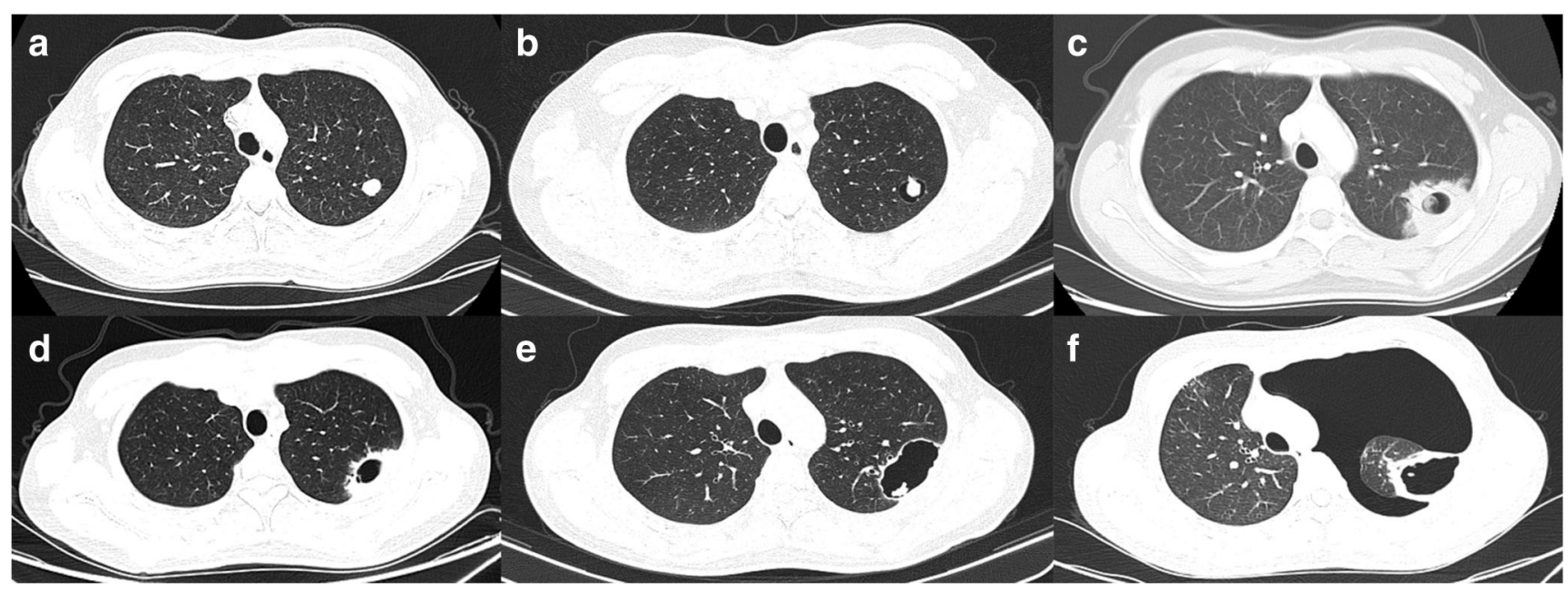

Fig. 3 Typical development of pneumothorax after apatinib treatment in an osteosarcoma patient. Computed tomography scans were obtained a at treatment initiation, b 1 month, c 2 months, $\mathbf{d} 3$ months, e 5 months, and f 6 months after treatment 
Consent to participate Formal consent was not required for this type of study.

\section{Consent for publication Not applicable.}

Availability of data and material All data generated or analysed during this study are included in this published article.

\section{Code availability Not applicable.}

Open Access This article is licensed under a Creative Commons Attribution 4.0 International License, which permits use, sharing, adaptation, distribution and reproduction in any medium or format, as long as you give appropriate credit to the original author(s) and the source, provide a link to the Creative Commons licence, and indicate if changes were made. The images or other third party material in this article are included in the article's Creative Commons licence, unless indicated otherwise in a credit line to the material. If material is not included in the article's Creative Commons licence and your intended use is not permitted by statutory regulation or exceeds the permitted use, you will need to obtain permission directly from the copyright holder. To view a copy of this licence, visit http://creativecommons.org/licenses/by/4.0/.

\section{References}

1. Bourcier K, Le Cesne A, Tselikas L et al (2019) Basic knowledge in soft tissue sarcoma. Cardiovasc Intervent Radiol 42:1255-1261

2. Biermann JS, Chow W, Reed DR, Lucas D, Adkins DR, Agulnik M, Benjamin RS, Brigman B, Budd GT, Curry WT, Didwania A, Fabbri N, Hornicek FJ, Kuechle JB, Lindskog D, Mayerson J, McGarry S, Million L, Morris CD, Movva S, O'Donnell RJ, Randall RL, Rose P, Santana VM, Satcher RL, Schwartz H, Siegel HJ, Thornton K, Villalobos V, Bergman MA, Scavone JL (2017) NCCN guidelines insights: bone Cancer, version 2.2017. J Natl Compr Cancer Netw 15:155-167

3. Von Mehren M, Randall RL, Benjamin RS et al (2018) Soft Tissue Sarcoma, Version 2.2018, NCCN clinical practice guidelines in oncology. J Natl Compr Cancer Netw 16:536-563

4. Oncology SCoCA-CACSoC (2016) Chinese expert consensus on diagnosis and treatment of soft tissue sarcomas (version 2015). Zhonghua Zhong Liu Za Zhi 38:310-320

5. Jo VY, Doyle LA (2016) Refinements in sarcoma classification in the current 2013 World Health Organization classification of Tumours of soft tissue and bone. Surg Oncol Clin N Am 25:621-643

6. Singhi EK, Moore DC, Muslimani A (2018) Metastatic soft tissue sarcomas: a review of treatment and new pharmacotherapies. P T 43:410-429

7. Ritter J, Bielack SS (2010) Osteosarcoma. Ann Oncol 21:320-325

8. Ferrari S, Ruggieri P, Cefalo G, Tamburini A, Capanna R, Fagioli F, Comandone A, Bertulli R, Bisogno G, Palmerini E, Alberghini M, Parafioriti A, Linari A, Picci P, Bacci G (2012) Neoadjuvant chemotherapy with methotrexate, cisplatin, and doxorubicin with or without ifosfamide in nonmetastatic osteosarcoma of the extremity: an Italian sarcoma group trial ISG/OS-1. J Clin Oncol 30:2112-2118

9. Judson I, Verweij J, Gelderblom H, Hartmann JT, Schöffski P, Blay JY, Kerst JM, Sufliarsky J, Whelan J, Hohenberger P, KrarupHansen A, Alcindor T, Marreaud S, Litière S, Hermans C, Fisher C, Hogendoorn PC, dei Tos A, van der Graaf W, European Organisation and Treatment of Cancer Soft Tissue and Bone Sarcoma Group (2014) Doxorubicin alone versus intensified doxorubicin plus ifosfamide for first-line treatment of advanced or metastatic soft-tissue sarcoma: a randomised controlled phase 3 trial. Lancet Oncol 15:415-423

10. Miyamoto S, Kakutani S, Sato Y, Hanashi A, Kinoshita Y, Ishikawa A (2018) Drug review: Pazopanib. Jpn J Clin Oncol 48:503-513

11. Xie L, Ji T, Guo W (2017) Anti-angiogenesis target therapy for advanced osteosarcoma. Oncol Rep 38:625-636

12. Duffaud F, Mir O, Boudou-Rouquette P et al (2018) Efficacy and safety of regorafenib in adult patients with metastatic osteosarcoma: a non-comparative, randomised, double-blind, placebo-controlled, phase 2 study. Lancet Oncol 20:120-133

13. Tian S, Quan H, Xie C et al (2011) YN968D1 is a novel and selective inhibitor of vascular endothelial growth factor receptor-2 tyrosine kinase with potent activity in vitro and in vivo. Cancer Sci 102:1374-1380

14. Scott LJ (2018) Apatinib: a review in advanced gastric Cancer and other advanced cancers. Drugs 78:747-758

15. Shen G, Zheng F, Ren D et al (2018) Anlotinib: a novel multitargeting tyrosine kinase inhibitor in clinical development. J Hematol Oncol 11:120

16. Zhou M, Chen X, Zhang H et al (2019) China National Medical Products Administration approval summary: anlotinib for the treatment of advanced non-small cell lung cancer after two lines of chemotherapy. Cancer Commun 39:36

17. Tian Z, Gu Z, Wang X et al (2019) Efficacy and safety of apatinib in treatment of osteosarcoma after failed standard multimodal therapy. Medicine 98:e15650

18. Tian Z, Wang X, Liu Z, Wang J, Yao W, Zhao Y, Gao S, Zhang P, Ge H (2019) Safety and efficacy of combination therapy with apatinib and doxorubicin in metastatic soft tissue sarcomas: an observational study from multiple institutions. Cancer Manag Res 11: 5293-5300

19. Chi Y, Fang Z, Hong X, Yao Y, Sun P, Wang G, du F, Sun Y, Wu Q, Qu G, Wang S, Song J, Yu J, Lu Y, Zhu X, Niu X, He Z, Wang J, Yu H, Cai J (2018) Safety and efficacy of Anlotinib, a multikinase angiogenesis inhibitor, in patients with refractory metastatic softtissue sarcoma. Clin Cancer Res 24:5233-5238

20. Wilding CP, Elms ML, Judson I, Tan AC, Jones RL, Huang PH (2019) The landscape of tyrosine kinase inhibitors in sarcomas: looking beyond pazopanib. Expert Rev Anticancer Ther 19:971991

21. Rongyuan Z, Zhiming W, Xi G et al (2019) The short-term efficacy and safety of anlotinib combined with chemotherapy in treatment of advanced soft tissue sarcoma. Chin J Clin Med 3:378-381

22. Xie L, Xu J, Sun X, Tang X, Yan T, Yang R, Guo W (2019) Apatinib for advanced osteosarcoma after failure of standard multimodal therapy: an open label phase II clinical trial. Oncologist 24: e542-e550

23. Weitao Y, Fangxing W, Qiqing C et al (2019) Efficacy and safety of apatinib in advanced sarcoma. Anti-Cancer Drugs 30:749-756

24. Li SL, Yang QK, Chen P, Zheng K, Wang W, Pei Y, Zhang XJ (2019) Clinical efficacy and safety of apatinib combined with chemotherapy for osteosarcoma and soft tissue sarcoma with pulmonary metastasis. Zhonghua Zhong Liu Za Zhi 41:309-314

25. Zheng K, Xu M, Wang L et al (2018) Efficacy and safety of apatinib in advance osteosarcoma with pulmonary metastases: a singlecenter observational study. Medicine 97:e11734

26. Grignani G, Palmerini E, Dileo P et al (2012) A phase II trial of sorafenib in relapsed and unresectable high-grade osteosarcoma after failure of standard multimodal therapy: an Italian sarcoma group study. Ann Oncol 23:508-516

27. Mao W-f, M-h S, Gao P-t et al (2012) The important roles of RET, VEGFR2 and the RAF/MEK/ERK pathway in cancer treatment with sorafenib. Acta Pharmacol Sin 33:1311-1318

28. Xie C, Wan X, Quan H et al (2018) Preclinical characterization of anlotinib, a highly potent and selective vascular endothelial growth factor receptor-2 inhibitor. Cancer Sci 109:1207-1219 
29. Wilhelm SM, Dumas J, Adnane L, Lynch M, Carter CA, Schütz G, Thierauch KH, Zopf D (2011) Regorafenib (BAY 73-4506): a new oral multikinase inhibitor of angiogenic, stromal and oncogenic receptor tyrosine kinases with potent preclinical antitumor activity. Int J Cancer 129:245-255

30. Wilhelm SM, Carter C, Tang L, Wilkie D, McNabola A, Rong H, Chen C, Zhang X, Vincent P, McHugh M, Cao Y, Shujath J, Gawlak S, Eveleigh D, Rowley B, Liu L, Adnane L, Lynch M, Auclair D, Taylor I, Gedrich R, Voznesensky A, Riedl B, Post LE, Bollag G, Trail PA (2004) BAY 43-9006 exhibits broad spectrum oral antitumor activity and targets the RAF/MEK/ERK pathway and receptor tyrosine kinases involved in tumor progression and angiogenesis. Cancer Res 64:7099-7109
31. Liao Z, Li F, Zhang C et al (2019) Phase II trial of VEGFR2 inhibitor apatinib for metastatic sarcoma: focus on efficacy and safety. Exp Mol Med 51:24

32. Xie L, Guo W, Wang Y et al (2018) Apatinib for advanced sarcoma: results from multiple institutions' off-label use in China. BMC Cancer 18:396

33. Li J, Qin S, Xu J, Guo W, Xiong J, Bai Y, Sun G, Yang Y, Wang L, Xu N, Cheng Y, Wang Z, Zheng L, Tao M, Zhu X, Ji D, Liu X, Yu H (2013) Apatinib for chemotherapy-refractory advanced metastatic gastric Cancer: results from a randomized, placebo-controlled, parallel-arm, phase II trial. J Clin Oncol 31:3219-3225

Publisher's note Springer Nature remains neutral with regard to jurisdictional claims in published maps and institutional affiliations. 